Document downloaded from:

http://hdl.handle.net/10251/66518

This paper must be cited as:

García Sanjuan, F.; Jaén Martínez, FJ.; Catalá Bolós, A. (2015). Augmented Tangible Surfaces to Support Cognitive Games for Ageing People. En Ambient Intelligence - Software and Applications. Springer. 263-271. doi:10.1007/978-3-319-19695-4_27.

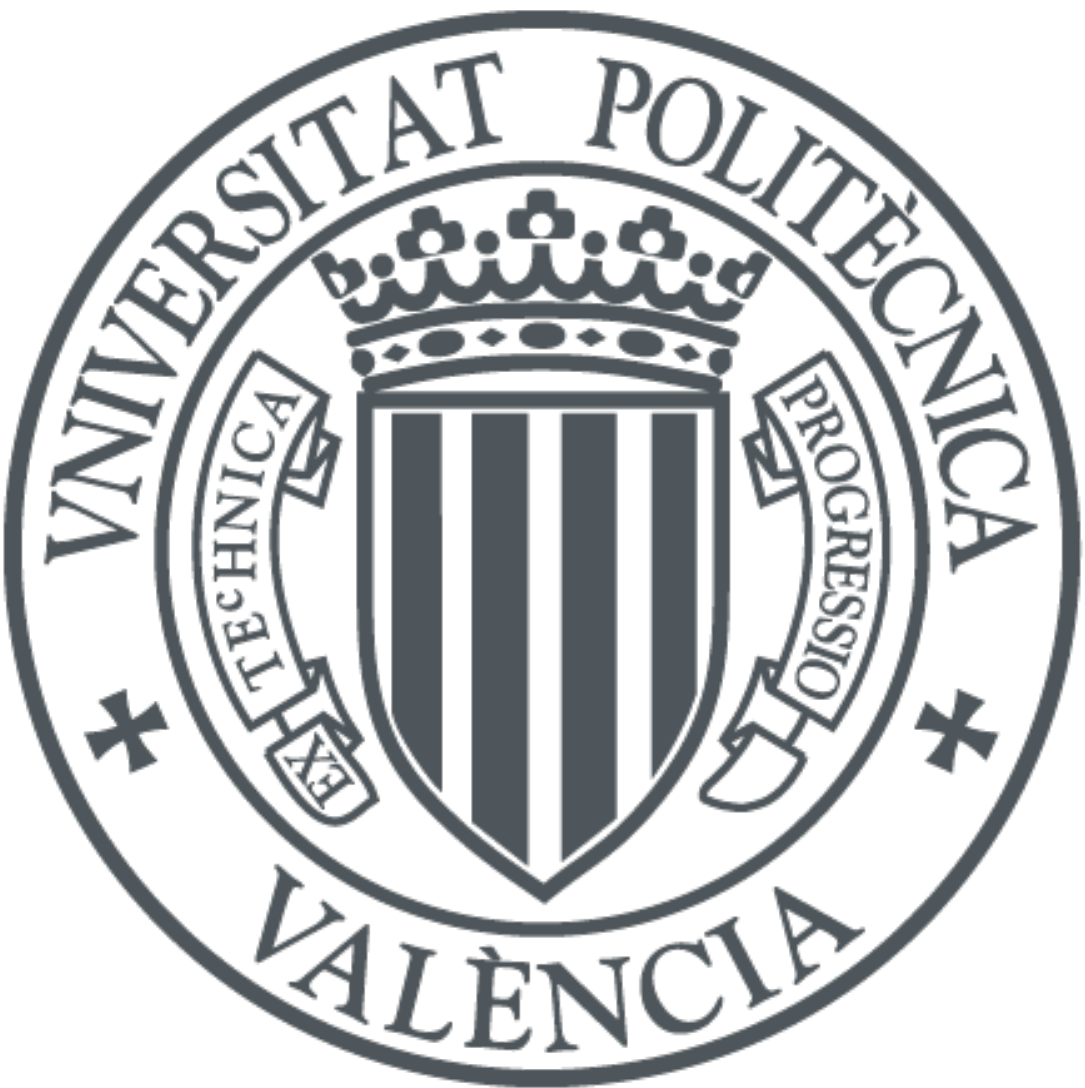

The final publication is available at

http://link.springer.com/chapter/10.1007/978-3-319-19695-4_27

Copyright Springer

Additional Information

The final publication is available at Springer via http://dx.doi.org/10.1007/978-3-319-196954227 


\title{
Augmented Tangible Surfaces to Support Cognitive Games for Ageing People
}

\author{
Fernando Garcia-Sanjuan, Javier Jaen, Alejandro Catala \\ Grupo ISSI, Departamento de Sistemas Informáticos y Computación, \\ Universitat Politècnica de València, Camí de Vera S/N, 46022 Valencia, Spain \\ \{fegarcia, fjaen, acatala\}@dsic.upv.es
}

\begin{abstract}
The continuous and rapidly increasing elderly population requires a revision of technology design in order to devise systems usable and meaningful for this social group. Most applications for ageing people are built to provide supporting services, taking into account the physical and cognitive abilities that decrease over time. However, this paper focuses on building technology to improve such capacities, or at least slow down their decline, through cognitive games. This is achieved by means of a digitally-augmented table-like surface that combines touch with tangible input for a more natural, intuitive, and appealing means of interaction. Its construction materials make it an affordable device likely to be used in retirement homes in the context of therapeutic activities, and its form factor enables a versatile, quick, and scalable configuration, as well as a socializing experience.
\end{abstract}

Keywords: gerontechnology, cognitive games, socialization, collaboration, Tangible User Interfaces (TUI), surfaces

\section{Introduction}

The number of ageing people in the European Union is fiercely increasing. According to Eurostat's statistics ${ }^{1}$, EU's elderly population is expected to rise from $17.9 \%$ in 2012 to $28.1 \%$ by the year 2050 due to the average increase of life expectancy and the continuous decline in birth rates. This growth will require adapting existing technological services and creating new ones for this group of people [1].

The idea of ageing people and technology being incompatible is a cliché, as it has been already proven in the literature. It is not true that the elderly have not the capacity or the will to learn and use new technologies. They do have the ability, although not necessarily the necessity [2]. It would appear that, traditionally, technological devices have been designed for youngsters, and neither their purpose nor interfaces are appealing to ageing people. In fact, a study conducted by Fisk et al. [3] concluded that more than half of the problems that elders experience with technology were associated with usability issues. In particular, the design of input/output devices and user interfaces is critical because they interact with the user's perceptual and sensorial

$1 \mathrm{http} / / /$ ec.europa.eu/eurostat/statistics-explained/index.php/Population_structure_and_ageing

adfa, p. 1, 2011.

(C) Springer-Verlag Berlin Heidelberg 2011 
system, which, at certain age, experience some changes that may have a negative impact on usability [3,4]. Examples of these changes are decrease of visual and acoustic capacities, touch- and movement-related issues (such as arthritis, tremors, walking problems, etc.), and a reduction of some cognitive capacities [5].

Traditionally, the most common ways of interacting with computers were using mouse and keyboard, but these present severe usability issues that can cause the elderly to be reluctant to engage with technology [6]. Direct contact via touch interfaces, instead, has shown to be more adequate to ageing users since these interfaces present less cognitive load and less spatial demand, and many efforts are being made as of late in order to create more intuitive user experiences using this kind of input devices [7]. Furthermore, Torres [6] proposes to devise alternative ways of performing input, for example, via tangible interfaces, which are typically referred as Tangible User Interfaces (TUIs) [8]. These offer spatial mapping, input/output unification, and the support of trial-and-error actions that can exploit innate spatial and tactile abilities; and have already been used successfully in cognitive training activities [9].

The present work contributes to the field with a TUI prototype in the form of a table-like surface aiming at building games for the elderly to train their cognitive abilities (see Fig. 1). It intends to be usable by providing a scalable and versatile means of configuration for both ageing people and the therapists who design the games, and by enabling a more natural interaction through tangible manipulations along with fully supporting touch interactions. Another important purpose of our proposed infrastructure is to foster socialization and the training of cognitive abilities that can improve elders' quality of life. The rest of the document is structured as follows: First, related work on technology for the elderly is described; then, our augmented tangible surface is presented; section 4 explains how our prototype could be used to build games for elders' cognitive training; and, finally, future work and conclusions are drawn.

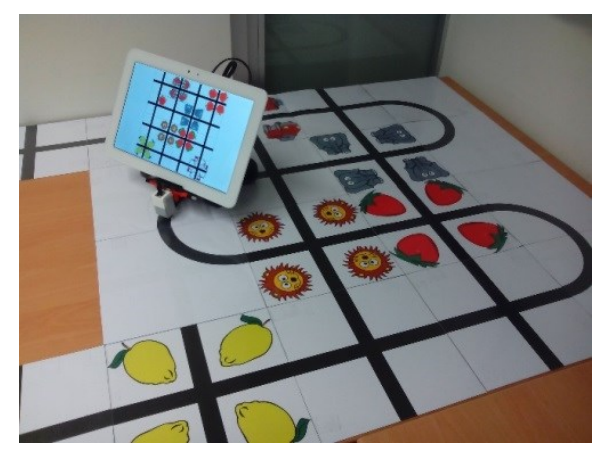

Fig. 1. Example of a game running on the surface

\section{$2 \quad$ Related Work}

Many research works have proposed technology to help ageing people deal with age-related problems in respect of physical and cognitive capacities. Some of them, in the form of assistive robots or mobile applications (e.g., [10-12]), offer services that 
improve the quality of life of the elderly and enhance their independence. However, they are often devised as aiding tools and not as therapeutic mechanisms to reduce the negative impact of their decreasing capacities. Besides, they are usually designed as private devices, omitting socialization, despite ageing people seem to assign a high value to socialization and they even report being against technology when it replaces face-to-face interactions [13]. In terms of socialization, there have been efforts using robots, called assistive social robots [14], focusing on improving socialization between themselves and ageing users. However, they do not intend to foster human-tohuman socialization. In fact, some authors have expressed their concerns about these technologies incrementing social isolation [15].

In addition to robots, other works propose the use of digital games (a.k.a. cognitive games) that stimulate the previously mentioned decreasing cognitive abilities, and also foster socialization (e.g., $[4,16,17])$. In this sense, playing represents an advantageous way to engage elder users both cognitively and socially [5]. There are many references in the literature stressing the benefits of playing videogames for the elderly. They have been proved to decrease reaction times $[18,19]$, and improve quality of life, self-confidence, and cognitive skills (these two showing a positive correlation) [6]. Moreover, Whitcomb [16] analyzed how ageing people played a series of videogames, and observed that they increased social interaction and perceptual-motor capacities (eye-hand coordination, dexterity, fine motor ability, and a reduction of the reaction time). Also, although the author did not explicitly study how videogames affected cognitive capacities, the study detected a positive effect of videogames on information processing, reading, comprehension, and memory.

Interaction design for videogames targeting elder people is a critical dimension to be considered. Whitcomb [16] also enumerates several characteristics that make a videogame unsuitable for them, such as small-sized objects, rapid movements or reactions required, or the sound being inappropriate. In terms of interaction mechanisms, this study focused on computer games, which are mainly interacted through mouse and keyboard. However, other interaction mechanisms, as we discussed in the previous section, may be advantageous when considering ageing people. In this respect, authors such as Jung et al. [20] have explored other input/output devices, e.g. a Wii stick in a game to enhance general wellbeing (physical activity, self-esteem, affect, and level of solitude). However, in our opinion, this type of interaction should be considered with caution when the elderly are involved because it has been reported to produce physical lesions such as tendinitis (or Wiiitis as it has been called) [21]. Alternatively, Chiang et al. [22], through Kinect games, report elder users improving significantly their visual performance skills. Others, however, have taken advantage of the increasing popularization of handheld devices. MemoryLane [4], although not exactly a game, fosters reminiscence through a PDA application to create "memory stories" with pictures. Vasconcelos et al. present CogniPlay [17], a gaming platform running on tablets which includes several games to stimulate cognitive abilities, such as matching pairs to enhance short-term memory, and social interaction through competition. However, the consideration of small devices or elements that are designed to be used by a single user is clearly a step in the wrong direction when collaboration needs to be fostered. 
Our approach intends to merge touch interaction capabilities provided by handheld devices such as tablets but at the same time taking advantage of the natural and intuitive manipulations that physical (tangible) elements can bring. Moreover, by proposing a surface-like configuration with such affordable materials and devices, a costeffective public space can be built where elder players can all have a simultaneous and equal access to the game space which fosters collaboration.

\section{Designing the Prototype}

The prototype presented in this work aims at supporting collaborative therapeutic games for the elderly around physical tables. Current digital tabletop technology would indeed allow us to deliver fine-grained touch interactions and high-end visual representations, but fully interactive tabletops are still expensive, and their form factor complicates their mobility and scalability. Instead, we propose a cost-effective way of creating a surface by arranging physical tiles, which can form a table-like interactive 2D environment of any arbitrary topology anywhere on a flat terrain. The resulting surface becomes a public space where all users can collaborate in problem solving tasks, and therapists can design cognitive games, such as matching pairs to train shortterm memory, simply by handling the physical tiles, without any technological knowledge required. This type of surface is digitally augmented in order to provide richer features to the games. However, to decrease the decoupling between the physical and the digital space that would take place if the digital information was shown in a separate display held by the players, a tablet is instead mounted on a small mobile robot that moves through the physical surface displaying digital contents within the context of the physical space (see Fig. 1). With respect to the input mechanisms supported for the elder participants, they can both use touch contacts and gestures on the tablet, and interact directly with the physical surface by adding and removing tiles that have a specific digital behavior associated or by giving commands to the robot using special physical tags, hence providing a more natural and intuitive interaction.

Our augmented tangible surface consists of two major components, as can be seen in Fig. 2. The 2D surface can be constructed by arranging several $20 \mathrm{~cm} \mathrm{x} 20 \mathrm{~cm}$ tiles following any desired flat configuration. Each tile has a number of black lines which allow the robot to move in the physical space in different directions by following them. The lines may represent a crossroads for the robot to choose which direction to take, or a specific path such as a curve. Depending on the game, each tile may also contain some drawings that make sense to the users in the context of the activity being developed. As Fig. 3 (left) depicts, each tile consists of a squared piece of paper with the path black lines and possibly the drawings, an RFID tag to be read by the robot when passes over it and which provides the tablet with digital information, another piece of paper with only the path printed (representing the back of the tile), and two pieces of plastic to protect it all.

The robot has been constructed using Lego ${ }^{\mathrm{TM}}$ Mindstorms ${ }^{\circledR}$ Ev3 and it has an Android tablet on it that serves as a rich colorful digital input/output device. Fig. 3 (right) shows the different components this robot is composed of (aside from the tablet). It 
has a color sensor that differentiates between black and white so it can follow the surface's black paths. Every time it reaches the center of a tile, its RFID reader situated on the bottom reads the tag embedded in the tile and sends its code to the tablet. This one contains the game logic, handles touch interaction, and sends the proper control commands to the robot via Bluetooth.

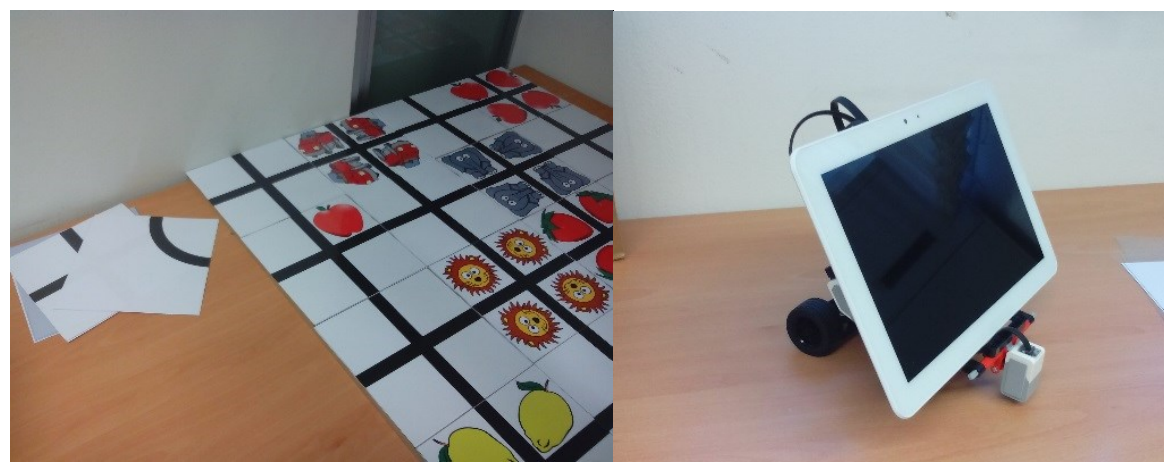

Fig. 2. The tangible surface's parts. On the left, the different tiles that compose the physical surface. On the right, the mobile robot that displays the digital content

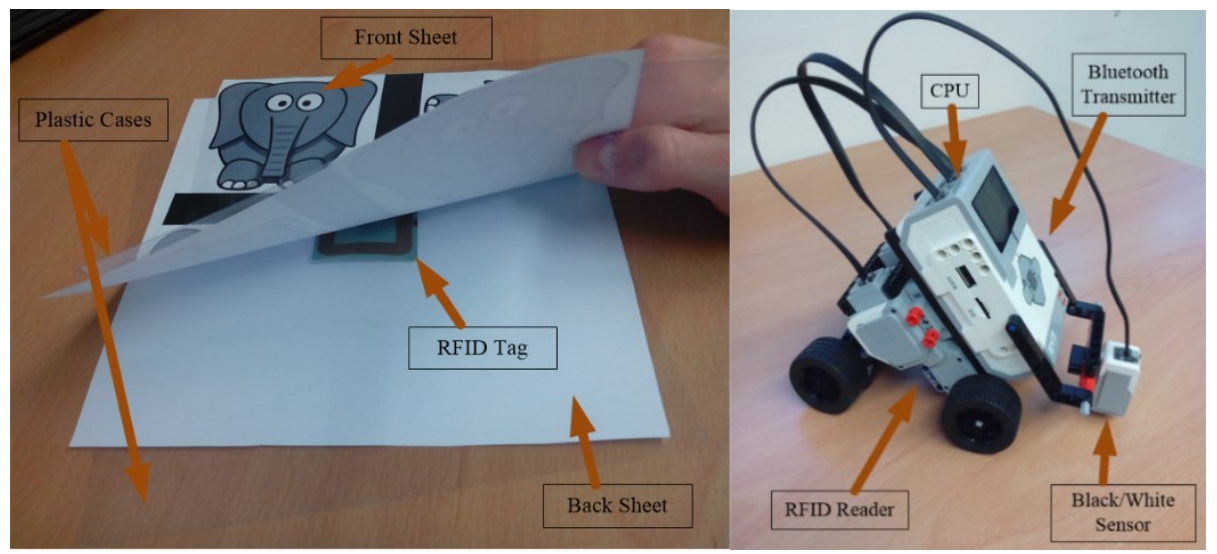

Fig. 3. Details of the tile (left) and the mobile robot (right)

The system allows several interaction modalities: Users can perform coarse-grained interactions by coupling and decoupling the tiles at will at runtime or by using command cards that are read by the robot's RFID reader. On the other hand, finer-grained interactions can also be achieved via touch contacts on the tablet. Since different tiles have distinct RFID codes, they can provide the game with different information, thereby removing the need of touch input, and leaving the tablet for display purposes only if this would be required. Fig. 4 shows an example of a touch interaction (left), where the user touches the tablet designing a path for the robot to follow, and of a tangible interaction (right), where the player physically "draws" the path in the surface by rearranging the tiles. 


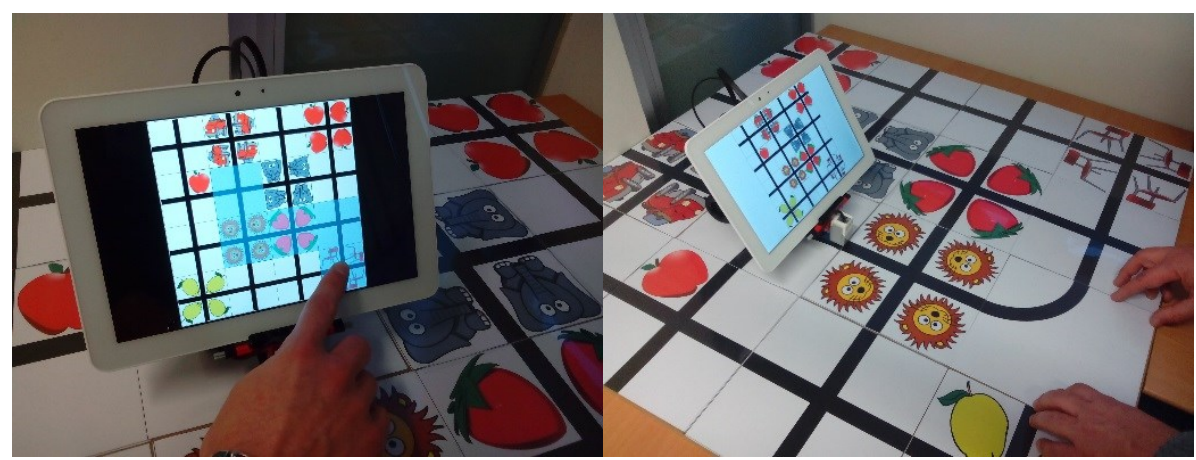

Fig. 4. Interaction modalities supported. Touch-based (left) and tangible (right)

\section{A Game to Stimulate Cognitive Abilities for the Elderly}

Ageing entails a diminution in some physical and cognitive capacities. Examples of these reduced capacities are short-term (working) memory, the ability to filter irrelevant information, divided attention, and visual-spatial attention [5]. This section exemplifies how the prototype described in this paper can be used to help training these capacities and fight their decline through developing cognitive games. An illustrative scenario of a game to improve short-term memory and divided attention is detailed next:

A therapist arranges several tiles containing pictures on the surface as depicted in Fig. 4 for the player to memorize. Then, the former turns over the tiles, removing the pictures and leaving only the crossing paths visible. At this moment, the tablet shows a target image and the user must devise a path from the robot position to the location where the displayed figure is. Then, using either touch or tangible interaction, the users draw the trajectory and the robot follows it. The system provides positive or negative feedback depending on the adequacy of the path defined to reach the proposed target. In additional iterations of the game the therapist also includes tiles showing a wrong direction sign to motivate the users to find alternative paths to the target avoiding these tiles.

Following Salthouse and Babcock's suggestions [23], both the speed of the robot and the rate at which the target elements are displayed should be reduced. According to Rogers [24], the reduction of the ability to filter irrelevant information affects the selective attention, which depends on the familiarity of the user with the presented objects. Taking this into account, the images to memorize should be easily recognizable by the participants. This game requires using divided attention because players must focus on remembering the location of the images and drawing paths at the same time. This training by itself, as stated by Rogers [24] enhances divided attention and improves making attention switches. Also, the remembrance of the objects' location and the creation of routes serve as a training of visual-spatial processes.

Another important aim of this platform is avoiding the dangers of social isolation that could provide a similar implementation where each user would hold a tablet. The 
intrinsic nature of our table-like surface enables collaborative scenarios where several people situate around the physical table and help one another find better pathways and/or simply discuss the game and the situation themselves.

\section{$5 \quad$ Conclusions and Future Work}

In this paper we presented a prototype of a digitally-augmented tangible surface aimed at constructing cognitive games for the elderly. Not only the table-like design fosters human-to-human socialization via collaboration but also the touch and tangible capabilities bring more natural and intuitive interactions that can appeal ageing users. Hence, the ultimate purpose of the present work is to design useful and usable technology for this special group.

The platform is built with cost-effective materials, and its design allows for a quick setup and a high versatility and scalability. We exemplified the use of the surface with a cognitive game to improve short-term memory and selective, divided, and visualspatial attention.

As future work, we intend to perform experiments with real users in order to test whether the tangible interaction offers any advantages with respect to digital (touch) both in configuring the layout of the game (i.e., the arrangement of the tiles) and in the problem solving phase (e.g., drawing a path for the robot to follow or giving it specific instructions at run time). Other future experiments will focus on the actual perceived usefulness and usability of the platform and on whether this system has any positive effect on the already enumerated cognitive capacities meant to be stimulated.

\section{Acknowledgements}

This work received financial support from Spanish Ministry of Economy and Competitiveness under the National Strategic Program of Research and Project TIN201020488, and from Universitat Politècnica de València under Project UPV-FE-2014-24. It is also supported by fellowships APOSTD/2013/013 and ACIF/2014/214 within the VALi $+d$ program from Conselleria d'Educació, Cultura i Esport (GVA).

\section{References}

1. Nunes, F., Silva, P.A., Abrantes, F.: Human-computer interaction and the older adult: an example using user research and personas. Proc. PETRA '10. pp. 49:1-49:8. ACM, New York (2010).

2. Durick, J., Robertson, T., Brereton, M., Vetere, F., Nansen, B.: Dispelling ageing myths in technology design. Proc. OzCHI '13. pp. 467-476. ACM, New York (2013).

3. Fisk, A.D., Rogers, W.A., Charness, N., Czaja, S.J., Sharit, J.: Designing for older adults: Principles and Creative Human Factors Approaches. CRC Press (2004).

4. Carthy, S.M., Sayers, H., Kevitt, P.M., McTear, M.: MemoryLane: Reminiscence for Older Adults. Proc. 1st Int. Workshop on Reminiscence Systems. pp. 22-27 (2009). 
5. Gamberini, L., Alcaniz, M., Barresi, G., Fabregat, M., Ibanez, F., Prontu, L.: Cognition, technology and games for the elderly: An introduction to ELDERGAMES Project. PsychNology J. 4, 285-308 (2006).

6. Torres, A.C.S.: Cognitive effects of video games on old people. Int. J. Disabil. Hum. Dev. $10,55-58(2011)$.

7. Loureiro, B., Rodrigues, R.: Multi-touch as a Natural User Interface for elders: A survey. 6th Iberian Conference on Information Systems and Technologies. pp. 1-6. IEEE (2011).

8. Ishii, H., Ullmer, B.: Tangible bits: towards seamless interfaces between people, bits and atoms. Proc. CHI '97. pp. 234-241. ACM, New York (1997).

9. Sharlin, E., Itoh, Y., Watson, B., Kitamura, Y., Sutphen, S., Liu, L., Kishino, F.: Spatial tangible user interfaces for cognitive assessment and training. In: Ijspeert, A.J., Murata, M., and Wakamiya, N. (eds.) Biologically Inspired Approaches to Advanced Information Technology. pp. 137-152. Springer Berlin Heidelberg (2004).

10. Montemerlo, M., Pineau, J., Roy, N., Thrun, S., Verma, V.: Experiences with a mobile robotic guide for the elderly. Proc. AAAI '02. pp. 587-592. AAAI, Menlo Park (2002).

11. Otjacques, B., Krier, M., Feltz, F., Ferring, D., Hoffmann, M.: Helping older people to manage their social activities at the retirement home. Proc. BCS-HCI '09. pp. 375-380. BCS, Swinton (2009).

12. Goodman, J., Brewster, S., Gray, P.: Older people, mobile devices and navigation. Proc. HCI '04, HCI and the older population workshop. pp. 13-14 (2004).

13. Eggermont, S., Vandebosch, H., Steyaert, S.: Towards the desired future of the elderly and ICT: Policy recommendations based on a dialogue with senior citizens. Poiesis Prax. 4, 199-217 (2006).

14. Broekens, J., Heerink, M., Rosendal, H.: Assistive social robots in elderly care: a review. Gerontechnology. 8, 94-103 (2009).

15. Sharkey, A., Sharkey, N.: Granny and the robots: Ethical issues in robot care for the elderly. Ethics Inf. Technol. 14, 27-40 (2012).

16. Whitcomb, G.R.: Computer games for the elderly. Proc. CQL '90. pp. 112-115. ACM, New York (1990).

17. Vasconcelos, A., Silva, P.A., Caseiro, J., Nunes, F., Teixeira, L.F.: Designing tablet-based games for seniors: the example of CogniPlay, a cognitive gaming platform. Proc. FnG '12. pp. 1-10. ACM, New York (2012).

18. Clark, J.E., Lanphear, A.K., Riddick, C.C.: The effects of videogame playing on the response selection processing of elderly adults. J. Gerontol. 42, 82-85 (1987).

19. Dustman, R.E., Emmerson, R.Y., Steinhaus, L.A., Shearer, D.E., Dustman, T.J.: The effects of videogame playing on neuropsychological performance of elderly individuals. J. Gerontol. 47, 168-171 (1992).

20. Jung, Y., Li, K.J., Janissa, N.S., Gladys, W.L.C., Lee, K.M.: Games for a Better Life: Effects of Playing Wii Games on the Well-Being of Seniors in a Long-Term Care Facility. Proc. IE '09. pp. 5:1-5:6. ACM, New York (2009).

21. Bonis, J.: Acute Wiiitis. N. Engl. J. Med. 353, 2431-2432 (2007).

22. Chiang, I.-T., Tsai, J.-C., Chen, S.-T.: Using Xbox 360 Kinect Games on Enhancing Visual Performance Skills on Institutionalized Older Adults with Wheelchairs. Proc. DIGITEL '12. pp. 263-267. IEEE (2012).

23. Salthouse, T.A., Babcock, R.L.: Decomposing adult age differences in working memory. Dev. Psychol. 27, 763-776 (1991).

24. Rogers, W.A.: Attention and aging. In: Park, D.C. and Schwarz, N. (eds.) Cognitive aging: A primer. pp. 57-73. Psychology Press, New York (2000). 